\title{
A Simplified Overview of the Current Non-Surgical Treat- ment of Brain Tumor Patients with Special Emphasis on Its Complications. A Much Needed Contemporary Review Especially for Neurosurgeons
}

\author{
Samim Akhtar, ${ }^{1}$ Abhishek Chaturbedi, ${ }^{2 *}$ \\ ${ }^{1}$ Consultant Oncologist, Department of Medical Oncology, Nepal \\ ${ }^{2}$ Consultant Neurosurgeon, Department of Surgical Oncology, Nepal
}

\begin{abstract}
Brain tumors are complex entities with facts as numerous as stars in our sky, uncountable. They exist and thus every treating physician and surgeon happens to come across it. We try to put major contemporary practices and facts in a single bucket from learned skills of neurology and oncology tradition and things we received as feedback from our patients in clinical settings. Purpose of this work is to refresh your knowledge and preparedness for seeing patients with brain cancer. Brain tumors are molecularly heterogeneous and their features depend upon size, site, and genetic make-up of tumor, aggressive growth, and adjacent structures. Complications and their management are very challenging. This article will comfortably sail you through various aspects of management of brain cancers.
\end{abstract}

Keywords: Brain tumor, Glioblastoma, Non surgical therapy, Complications

Running Title: current non-surgical treatment of brain tumor and its complications

\section{Introduction}

Brain Tumors are diverse special neoplasm's, distinct from other tumors of human body, due to their cellular origin, complexity, blood-brain barrier, confinement within sphere of skull and potential to encroach and damage important structures of brain. The presentation of brain tumor may be silent, subtle or bizarre. It is of prime importance for Neurologist, Neuro Surgeons and Oncologist to be prepared to tackle these effects in patients. This is a concise plain review for above health care providers who are involved in management of CNS (Central Nervous System) Neoplasm's. From last two centuries, surgical removal of brain tumor was primary intervention, and so it is today. Surgery alone does not always suffice tumors of brain with malignant nature, because its neo plastic activities continue beyond excision. The very special character of brain neoplasm is recurrence and this led to development of other modalities of treatment: radiotherapy, chemotherapy, targeted therapy etc. There are special issues with each therapy. Here we delineate the adverse effects and long-term complications of non-surgical therapies. To understand this, we should follow classification of brain tumors.
Quick Response Code:

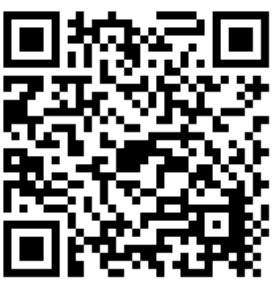

*Corresponding author: Abhishek Chaturbedi, MBBS, MS in Neurosurgery, Neurosurgery Fellowships (USA), Post-Doctoral Research Scholar (USA), Head of Neuroscience Service, Department of Surgical Oncology, Nepal Cancer Hospital, Satdobato-Godawari Road, Lalitpur, Nepal

Received: 28 June, 2021

Published: 12 July, 2021

Citation: Akhtar S, Chaturbedi A. A Simplified Overview of the Current Non-Surgical Treatment of Brain Tumor Patients with Special Emphasis on Its Complications. A Much Needed Contemporary Review Especially for Neurosurgeons. SOJ Neuro Neurosci. 2021;1(2):1-7. DOI: 10.53902/SOJNN.2021.01.000507 
Broadly brain tumors are grouped as Primary and Metastatic. American Association of Neurological Surgeons

Further Primary tumor is of glial and non-glial cells. Metastatic tumors are spread from other organs (Table 1).

\section{WHO classification of Brain Neoplasm}

It is much widely accepted based upon the character of tumor and different treatment options for individual group (Table 2 \& Table 3).

Table 1: Difference between primary and secondary GBM (Glioblastoma Multiforme).

\begin{tabular}{|l|l|}
\hline \multicolumn{1}{|c|}{ Primary GBM } & \multicolumn{1}{c|}{ Secondary GBM } \\
\hline$>90 \%$ GBM are primary & Others are secondary \\
\hline De novo GBM formation & Progression from LGG (Low Grade Glioma) to HGG (High Grade Glioma) \\
\hline Younger patient (<40 years) & Older patients ( $>55$ years) \\
\hline Minimal duration from symptoms to diagnosis (3 months) & Indolent clinical course as tumor progress from low grade to high grade \\
\hline EGFR amplification or over expression ( $>40 \%)$ & TP53 mutation (60\%) in Secondary GBM \\
\hline PTEN Deletion $(15-40 \%)$ & IDH1 and IDH2 mutation common in Grade II/III glioma \\
\hline LOH of Chromosome 10q (80\%) & 1p19q codeletion seen in Oligodendroglioma progressing to GBM \\
\hline$>90 \%$ GBM are primary & Others are secondary \\
\hline Life span 6-9 months & Life span 1-1.5 years \\
\hline
\end{tabular}

EGFR: Epidermal Growth Factor Receptor; PTEN: Phosphatase and Tens in homolog; IDH: Isocitrate dehydrogenase; LOH: Loss of heterozygosity

Table 2: Characteristics of various World Health Organization (WHO) grading of glioma.

\begin{tabular}{|l|l|l|l|}
\hline \multicolumn{1}{|c|}{ WHO Grade } & \multicolumn{1}{c|}{ Survival in years } & \multicolumn{1}{c|}{ MRI characteristics } & \multicolumn{1}{c|}{ Histological hallmark } \\
\hline $\begin{array}{l}\text { Grade I } \\
\text { (Pilocytic Astrocytoma) }\end{array}$ & $8-10$ & No mass effect, no enhancement & Cytological atypia present \\
\hline $\begin{array}{l}\text { Grade II } \\
\text { (Diffuse Astrocytoma) }\end{array}$ & $7-8$ & $\begin{array}{l}\text { mass effect present, no enhance- } \\
\text { ment }\end{array}$ & nuclear atypia present \\
\hline $\begin{array}{l}\text { Grade III } \\
\text { (Anaplastic Astrocytoma) }\end{array}$ & $2-3$ & Complex contrast enhancement & Anaplasia and mitotic activity \\
\hline $\begin{array}{l}\text { Grade IV } \\
\text { (Glioblastoma) }\end{array}$ & 1 & $\begin{array}{l}\text { Ring enhancement with necrotic } \\
\text { center }\end{array}$ & Neovascularization and necrosis \\
\hline
\end{tabular}

MRI, Magnetic Resonance Imaging

Table 3: Clinical presentation of patient with CNS neoplasm.

$\begin{array}{ll}\text { - } & \text { Headache } \\ \text { - } & \text { Decreased consciousness } \\ \text { - } & \text { Dysarthria/ speech problem } \\ & \text { Hemiparesis/ hemiplegia }\end{array}$

The success of new drug and treatment methods are evaluated and explained in terms of overall survival (OS) and progression free survival (PFS) in relation to comparisons of time in months or weeks. But these measures do not include the quality of life and co morbidity a patient sustains between PFS and OS. ${ }^{1}$ The initial clinical presentation (symptoms and signs) of brain tumor patient is important. More important is the sign, symptoms and adverse effects presented during initiation of any treatment, revising a chemotherapy plan, changing treatment modality and stopping the treatment. The treating Neuro surgeons, Neurologists and Oncologist should

$\begin{array}{ll}\text { - } & \text { Vomiting } \\ \text { - } & \text { Seizure } \\ \text { - } & \text { Visual problems } \\ & \text { Gait disturbances etc. }\end{array}$

be vigilant and be ready to deal with any unprecedented event. It means the doctor has to keep in mind chronologically all "disease and treatment" events of a brain tumor patient.

Methyl Guanine Methyl Transferase (MGMT),Isocitrate dehydrogenase-1 (IDH 1) mutation and 1p19q co-deletion tumor has good prognosis. ${ }^{2}$ Patient's age more than 40 years, poor performance score, tumor size $\geq 6 \mathrm{~cm}$, prominent neurological deficit, tumor crossing midline of brain are to be remembered as poor prognostic factors (Figure 1).

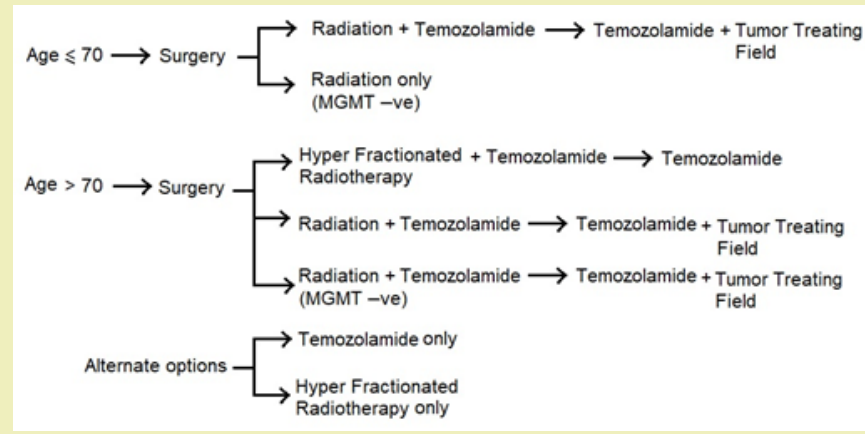

Figure 1: Simplified Treatment strategy from NCCN (National Comprehensive Cancer Network) Clinical Guideline 2020 . $^{3}$ 


\section{Radiotherapy}

Radiotherapy is important because it helps in controlling growth of tumor and improves survival duration. It has adverse effect of brain tissue necrosis. Generally, 56 to 60Gy is given. ('Gy' means 'Gray', a unit of quantification of radiation energy absorbed by 1 gram of body's tissue or mass. One Gray equals to 100 rads that is equivalent to 1Joule energy per kilogram. It has replaced the older measurement unit "rad"). ${ }^{4,5}$ Radiation is applied in fractionated doses. ${ }^{6}$ Radiation induced brain damage is grouped as

Acute: within days to weeks

Early delayed: within weeks to months

Late delayed: within months to years. ${ }^{7}$

Acute and early delayed radiation injury: Acute and early delayed injury happens due to damage to endothelium and blood brain barrier. It results in edema. It is reversible and cures spontaneously. ${ }^{8}$

Patient presents with headache vomiting seizures and altered level of consciousness in acute phase. It will be difficult to distinguish between baseline vasogenic edema due to original tumor and radiation induced brain edema in early injury. In the early delayed injury, brain edema near the irradiated border may be accompanied by temporary demyelization. It appears as new area of enhancement, near the margin of tumor. Patient may present with focal neurological deficit. ${ }^{9}$ Late delayed radiation injury is caused by permanent damage to vascular and glial cells. Gliosis occurs resulting progressive white matter necrosis. Loss of brain matter is seen. Radiation induced micro angiopathy and vasculopathy can lead to carotid artery stenosis. Carotid artery stenosis is rare complication but it can occur in highly vascular tumor like Glioblastoma. Ischemic stroke is a common presentation in Carotid stenosis but an unsuspecting highly vascular glioblastoma may be underlying cause. Thus, we need to be careful while making diagnosis based on radiology scans (Figure 2). ${ }^{10}$

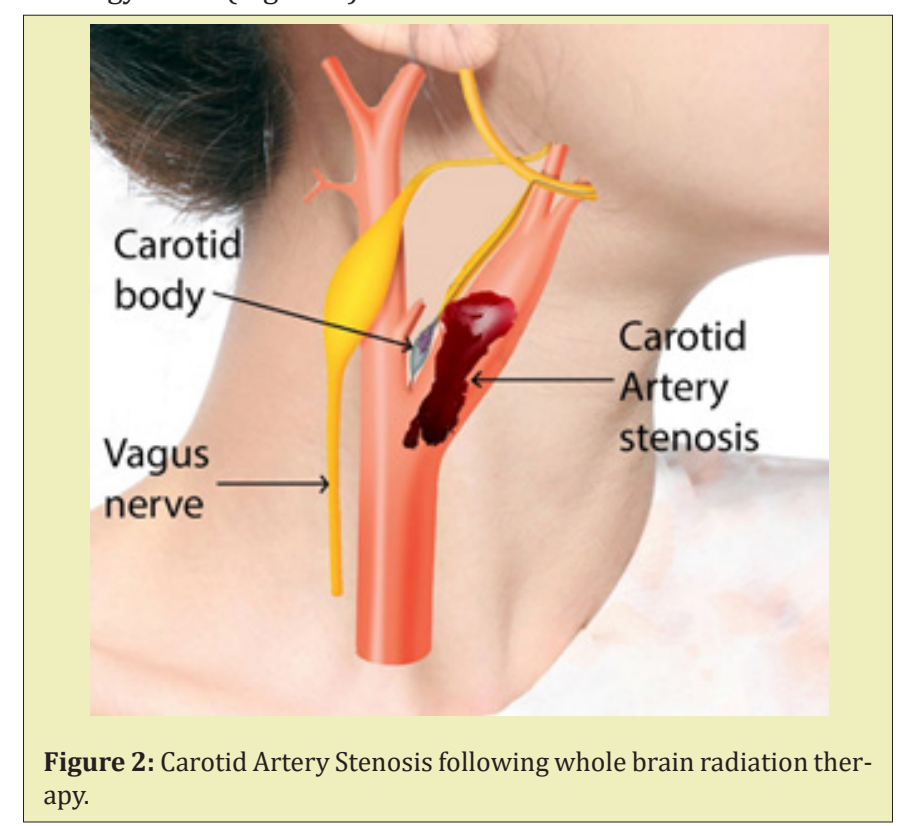

Radiation induced leukoencephalopathy occurs due to injury to white matter of brain without direct necrosis. Mostly patient who receive whole brain irradiation show progressive leukoencephalopathy within 6 months. ${ }^{11}$ Patient presents with deterioration of higher mental function after months though he may be asymptomatic initially (Figure 3). Sometimes treatment with anti VEGF (Vascular Endothelial Growth Factor) like Bevacizumab can lead to progressive multifocal leukoencephalopathy (PML). The image is confluent, symmetric and progressive in per ventricular white matter. It is difficult to identify this white matter changes. ${ }^{12}$ Laser Interstitial Thermal Therapy (LITT) is a thermo-coagulative therapy in which an optical fiber is used to cause necrosis and death of tumor cells. It is a novel treatment for recurrent Glioblastoma. It has adverse effect such as seizure in less than $2 \%$ of patients and transient hemi paresis $1 \%$. No mortality seen due to this treatment. It is one option for recurrent Glioblastoma and also primary disease. Chemotherapy is given after LITT treatment. ${ }^{13}$

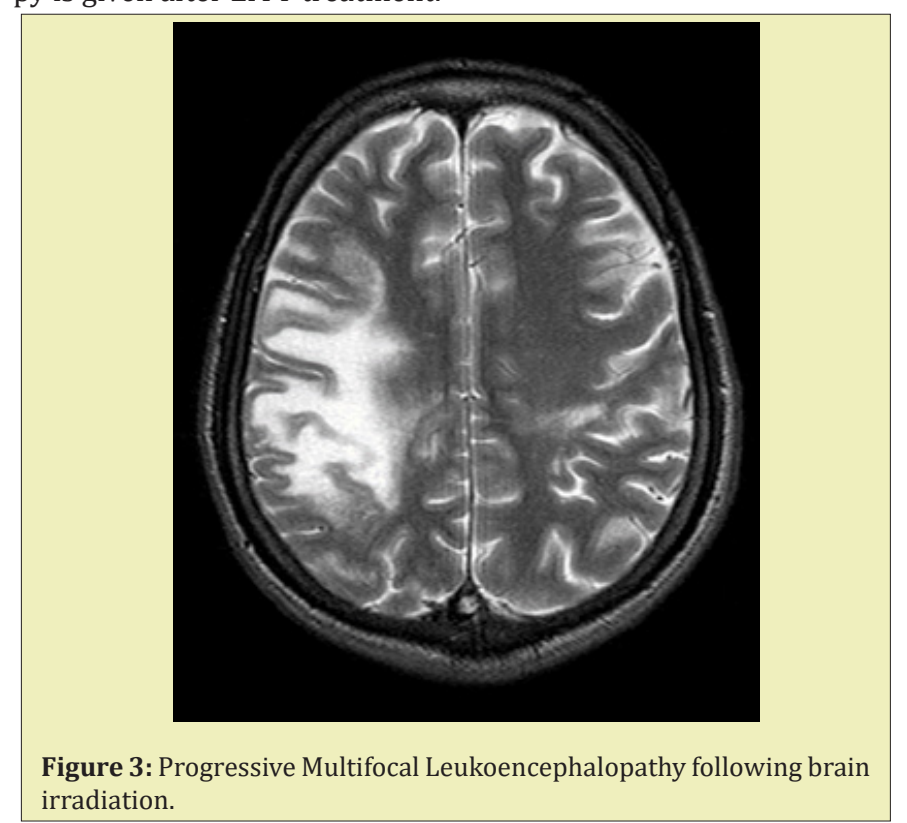

\section{Chemotherapy}

Temozolomide, an alkylating agent, is the standard chemotherapy currently used in treatment of Glioblastoma. The standard dose, dose dense and metronomic dose of Temozolomide, studied across by different researchers has mixed results. Dose dense Temozolomide has no significantly good prognosis than the standard dose. Few researches have shown metronomic regimen has better control of disease. Despite that, more than half of patients treated with Temozolomide have recurrence within 2 years. Common side effects of these drugs are alopecia, leucopenia, headache, vomiting etc. ${ }^{14,15}$ Side effects are waned off after discontinuation of treatment.

Chemotherapy has important role in brain tumors. A recent study conducted in Taiwan, concluded that the overall survival of patients, treated Bevacizumab (a monoclonal antibody) with chemotherapy Vincristine and low dose Carboplatin has significant overall survival as compared to those not treated with chemotherapy ( $p$ value $=0.006$ ). Vincristine is a anti angiogenic drug which is 
effective in glioblastoma. ${ }^{16}$ It is also given in combination with Lomustine.

Bevacizumab is a VEGF inhibitor, an antineoplastic monoclonal antibody which neutralizes the VEGF expression in cancer cells. It is helpful because glioblastoma is tumor with rich angiogenesis, blood vessels and neovascularization. Bevacizumab targets blood supply of glioblastoma. Common serious side effects of bevacizumab are bleeding poor wound healing. Some of our patients has presented with oral ulcers, pain and mucositis in oral cavity. In our experience, these adverse effects are treated/ well controlled with local analgesic, ointment and chlorhexidine mouth gargle. Diarrhea, abdominal bloating and loss of appetite were seen. These adverse reactions disappeared within few days to a week as soon as the therapy was postponed. ${ }^{17}$

\section{Immunotherapy}

Nivolumab is an immune check point under investigation. It is tried in combination with radiotherapy and Temozolomidein Glioblastoma. Currently immunotherapy is not showing any promise for excellent results. ${ }^{18}$ Majority of glioblastoma has significant PDL 1 (Programmed Death Ligand-1) expression, which can be exploited for immunotherapy. Various clinical researches has indicated some benefit of these therapies, yet vast dimension needs be explored. ${ }^{19}$

\section{Intracerebro ventricular chemotherapy}

Intrathecal Cytarabine or Methotrexate and Prednisolone is administered for treatment of CNS Lymphoma via Ommaya reservoir. Usually complications like headache, vomiting, fever (due to local infection), meningeal signs, ventriculitis, meningitis, CSF (cerebrospinal fluid) leakage, and somnolence may occur. We should be fluent in management of this dangerous complication. Steroids, antibiotics and septic precautions should be considered with best supportive care. Methotrexate can cause seizure and acute ventriculitis when administered through intrathecal route. ${ }^{20}$

\section{Targeted therapy}

Mammalian target of rapamycin (mTOR) inhibitor like Everolimus is approved for giant cell astrocytoma. Dose of Everolimus is decreased to alternate day if adverse drug reaction is seen. If symptoms increase then the drug can be held for a week. Commonly available medicine is used treat to treat adverse effects. ${ }^{21}$ Before introduction of targeted therapy, surgery was performed to majority of giant cell astrocytoma. Currently, we have option of targeted therapies for recurrent tumors. Stomatitis, infections, diarrhea, constipation anemia etc. are main adverse reaction of this drug.

\section{Kinase inhibitor}

Sorafenib is a multiple kinase inhibitor [Raf (Rapidly Accelerated Fibro sarcoma)/MEK (mitogen-activated protein kinase)/ERK (extracellular signal regulated kinase) and VEGF]. It is given twice a day; it has adverse events of skin and gastrointestinal toxicity. Sorafenib is an option of treatment, proven in clinical trials, when brain tumor is recurrent and patient cannot undergo repeated surgery or had already received brain irradiation. Common toxicities of this drug can be minimized. Patient can have benefit with corticosteroid. It has a beneficial effect on sorafenib toxicities. ${ }^{22}$

\section{Hand foot syndrome}

Hand foot syndrome a complication occurring in patients receiving chemotherapy and targeted therapy. An example of Sorafenib caused appearance of "Hand and Foot rash" within a week of beginning therapy (Figure 4). Characteristic tender, blanching hypo pigmented rash also known as Bergdorf's syndrome or Palmar-plantar Erythro-dysesthesia syndrome. The novel treatment of brain tumor has tolerable and manageable adverse effects. Toxicities can be managed with supportive treatment and general medicine. ${ }^{23}$

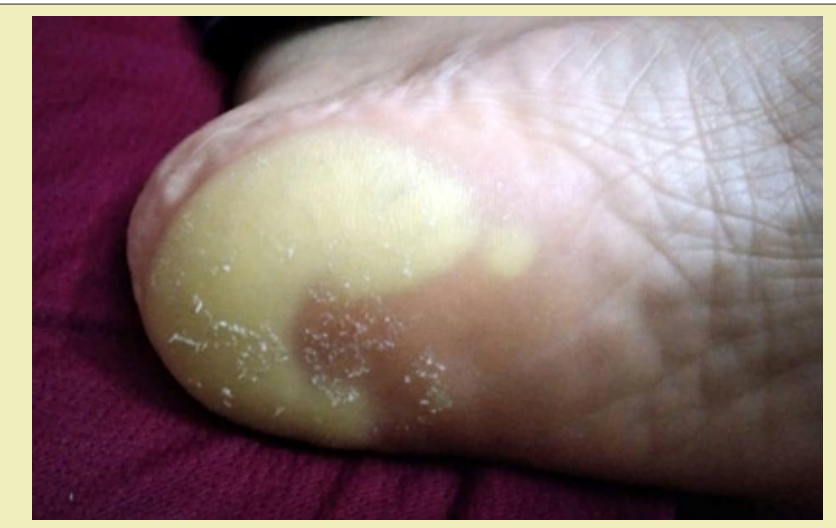

Figure 4: Hand Foot Syndrome in a recurrent meningioma patient.

\section{Targeted Therapy}

\section{Cyclin D 4/6 kinase inhibitors}

Abemaciclib or Palbociclib are suitable for treatment of metastatic brain tumors such as metastasis from breast cancer. May be used to treat primary brain tumor such as Glioblastoma to some extent. These inhibitsCDK4 and CDK6 (cyclin dependent kinase). Addition of Abemaciclib to Temozolomide was beneficial in rat experiments.Abemaciclib can cross blood brain barrier effectively and inhibits $\mathrm{Rb}$ (Retinoblastoma) protein in early G1 phase restricting cancer growth. Abemaciclib at lower doses has better CNS levels compared to Palbociclib. ${ }^{24}$ In our center, few patients receiving Palbociclib for metastatic brain cancer have reported of prolonged mild grade diarrhea, oral stomatitis loss of taste of food, for 3 to 4 weeks. Diarrhea was controlled using loperamide and oral mucositis was treated with oral analgesics, folic acid tablets and supplement vitamins. Few patients were advised to postpone treatment for a week. Their conditions improved quickly.

In radiological studies, Pseudo-progression mimics like a progression of disease. It looks like new enhancement and increase in size of treated brain tumor. Initially it looks like progression of tumor but decreases in size weeks later. This occurs after treatment with chemo radiation or Temozolomide. ${ }^{25}$ In Pseudo-response phenomena it looks rapid decrease in enhancement within a recently treated brain tumor. But, T2 imaging shows flair hyper intensity. Thus, we should cautiously interpret post treatment imaging results. ${ }^{26}$ 
Why it is important to follow up and test for therapy toxicity?

The rationale behind 3 weeks treatment and 1 week treatment free interval, and similar other chemo-planning, is for recovery from toxicity sustained by organs during days of active treatment.
It is time given for healing of normal structures. Because there is no standard protocol of chemotherapy for recurrent brain tumors at current time, different centers are undertaking clinical trials for search of more effective treatments (Table 4). ${ }^{27}$

Table 4: Proposed simplified patient investigation record for neuro oncology patients.

\begin{tabular}{|c|c|c|c|}
\hline Tests & Time & Evaluation & Time \\
\hline $\begin{array}{l}\text { Complete blood count with Dif- } \\
\text { ferential count } \\
\text { Platelet count }\end{array}$ & \multirow{2}{*}{$\begin{array}{l}\text { Base line before beginning } \\
\text { treatment } \\
\text { Then weekly for } 1 \text { month }\end{array}$} & Medical history & First time presentation \\
\hline Liver function tests & & $\begin{array}{l}\text { Clinical Examination including } \\
\text { skin, oral cavity, hand foot and } \\
\text { ano-genital }\end{array}$ & Examined at each visit \\
\hline Xray of Chest & Once Every 2 months & Neurological Examination & Examined at each visit \\
\hline USG Abdomen & Once in Every 2 months & Performance score & Estimated at each visit \\
\hline ECG & Monthly & \multirow[b]{2}{*}{$\begin{array}{l}\text { Grading of clinical and hema- } \\
\text { tological adverse effects/ re- } \\
\text { sults should be done National } \\
\text { Cancer Institute Common Ter- } \\
\text { minology Criteria for Adverse } \\
\text { Events (CTACE) }\end{array}$} & \multirow[b]{2}{*}{$\begin{array}{l}\text { Note down clinical in "Re- } \\
\text { marks" section of medical re- } \\
\text { cord assigned to specific date } \\
\text { in chronological order. }\end{array}$} \\
\hline Pregnancy test & As per sexual history of patient & & \\
\hline $\begin{array}{l}\text { Computerized Tomography } \\
\text { (CT) scan/ Magnetic Reso- } \\
\text { nance Imaging (MRI) Brain/ } \\
\text { Magnetic Resonance Spectros- } \\
\text { copy (MRS) }\end{array}$ & $\begin{array}{l}\text { (i) Before initiation of treat- } \\
\text { ment. } \\
\text { (ii)For evaluation in between } \\
\text { cycles of treatment. } \\
\text { (iii) After completion of treat- } \\
\text { ment }\end{array}$ & & \\
\hline
\end{tabular}

\section{Prognostic Implications}

Isocitrate dehydrogenate 1(IDH1) positivity is hopeful report, one should explain to patient, the good survival outcome related to it. Researchers in this path have found that IDH 1 mutant tumors have survival up to 3 times more than that of Wild IDH mutants. Younger age, TP53 mutation and IDH1 positive result is positive prognostic factors for GBM patients. ${ }^{28}$ Similarly, MGMT promoter methylation independent of IDH1 mutation has proven a powerful prognostic and predictive factor in glioblastomas patients. ${ }^{29} \mathrm{~Pa}-$ tients treated with Temozolomide with MGMT promoter has significant difference in survival, (increased overall survival) compared to those who received Temozolomide and radiation therapy but have no MGMT methylation. ${ }^{30}$ Lower grade gliomas those which harbor 1p19q chromosome co-deletion and IDH 1 mutation have good prognosis compared to other types of gliomas. The co-deletion of $1 \mathrm{p} 19 \mathrm{q}$ chromosome is more efficient in predicting good outcome than histological grading of brain tumor. IDH mutant tumors with no co-deletion of $1 \mathrm{p} 19 \mathrm{q}$ chromosome have shorter overall survival. ${ }^{31}$ EGF Rover expression is responsible for increased tumorigenicity via invasion and angiogenesis. It evades apoptosis. It confers bad prognosis in brain tumor. In one hand if EGFR promotes brain tumor, so do we have chances of treating CNS tumor by targeting EGFR points with targeted therapy? It is shown in recent research that EGFR mutation disappears after therapy and reappears after stopping therapy for brain tumor. Thus, taking advantage of EGFR mutation in context of brain tumors for treatment is controversial and debatable. More research is awaited in this arena (Table 5). ${ }^{32}$

Table 5: Molecular markers commonly tested for glioma patients.

\begin{tabular}{|l|l|l|}
\hline \multicolumn{1}{|c|}{ Specific molecular markers } & Prognostic implications & \multicolumn{1}{c|}{ Specific comments } \\
\hline IDH1 and IDH2 mutation & Protective factor & Longer survival (2 years) \\
\hline IDH wild type & Risk factor & Life expectancy similar to GBM (1 year) \\
\hline 1p19q deletion & Protective factor & Longer PFS and OS, better response to chemotherapy \\
\hline MGMT Methylation & Protective factor & Better response to Temozolomide \\
\hline TP53 mutation & Risk factor & $\begin{array}{l}\text { Progression to higher grade of glioma, seen in majority of sec- } \\
\text { ondary Glioblastoma }\end{array}$ \\
\hline EGFR vIII amplification & Risk factor & $\begin{array}{l}\text { Poor prognosis, but better response to Receptor Tyrosine Kinase } \\
\text { inhibitors }\end{array}$ \\
\hline PTEN mutation & Risk factor & Poor prognosis in pediatric Glioblastoma \\
\hline
\end{tabular}


Take away message for neurosurgeons and neurologists involved with neuro-oncology patients from Oncologist point of view

1. Malignant Glioma patients are heterogeneous genetically, even though they have similar histopathology.

2. Immunohistochemistry, epigenetic changes and molecular markers will soon take center stage in providing a tailor made, personalized treatment for patients with malignant glioma.

3. Request to Neuro- pathologist- Desired Molecular markers for

a) LGG-IDH mutation, 1p19q co-deletion.

b) HGG- MGMT methylation, EGFR vIII amplification/over expression, PTEN deletion, LOH of Chromosome 10

\section{Special imaging tools in glioma diagnosis}

a) MR Spectroscopy

b) MR Perfusion

c) PET Scan

d) SPECT(Single Photon Emission Computed Tomography) scan

e) PET (Positron Emission Tomography) and SPECT scan merged with CT or MRI brain

f) In general, above tests can give information regarding:

g) Low grade glioma Vs High Grade Glioma

h) Radiation induced necrosis Vs recurrence of tumor

i) Localization of stereotactic biopsy site in case of intra-tumoral heterogeneity

\section{Consequences of Long-Term Brain Cancer Survivors}

Most patients present with chronic fatigue syndrome, stroke, leukoencephalopathy, deteriorating mental function. Hypopituitarism, hypogonadism and Adrenocorticotropic hormone (ACTH) deficiency is also seen. Cerebral radiation necrosis, Strokelikemigraine attacks after radiation therapy (SMART), Radiation induced optic neuropathy are long term outcomes. Impaired hearing-auditory nerve damage, Chemotherapy Induced Peripheral Neuropathies (CIPN), Secondary neoplasms are seen. ${ }^{33}$ All of these patients should be given treatment, best support care, pain management. The long-term survival and complications depend on many factors. Genetic factors IDH mutations and MGMT promoter methylation are very important for long term survival. ${ }^{34}$

\section{Acknowledgements}

None.

\section{Funding}

This study has not received any funding and has no financial resources.

\section{Conflicts of Interests}

The authors declare no conflict of interest.

\section{References}

1. Armstrong TS, Dirve L, Arons D, et al. Glioma patient-reported outcome assessment in clinical care and research: a Response Assessment in Neuro-Oncology collaborative report. The Lancet Oncology. 2020;21(2):e97-e103.

2. Eckel Passow JE, Lachance DH, Molinaro AM, et al. Glioma Groups Based on 1p/19q, IDH, and TERT Promoter Mutations in Tumors. $N$ Engl J Med. 2015;372(26):2499-2508.

3. Nabors LB, Portnow J, Ahluwalia M, et al. Central Nervous System Cancers, Version 3.2020, NCCN Clinical Practice Guidelines in Oncology. JNCCN. 2020;18(11):1537-1570.

4. Lawrence YR, Li XA, el Naqa I, et al. Radiation dose-volume effects in the brain. International journal of radiation oncology, biology, physics. 2010;76(3 Suppl):S20-S27.

5. Minniti G, Filippi AR, Osti MF, et al. Radiation therapy for older patients with brain tumors. Radiation oncology (London, England). 2017;12(1):101.

6. Tan AC, Ashley DM, Lopez GY, et al. Management of glioblastoma: State of the art and future directions. CA: a cancer journal for clinicians. 2020;70(4):299-312.

7. Kessler AT, Bhatt AA. Brain tumour post-treatment imaging and treatment-related complications. Insights into imaging. 2018;9(6):10571075.

8. Greene Schloesser D, RobbinsME, Peiffer AM, et al. Radiation-induced brain injury: A review. Frontiers in oncology. 2012; 2(73).

9. Balentova S, Adamkov M. Molecular, Cellular and Functional Effects of Radiation-Induced Brain Injury: A Review. International journal of molecular sciences. 2015;16(11):27796-27815.

10. Xu Z, Luo B, Wang Q, et al. Glioblastoma multiform with ipsilateral carotid artery stenosis: carotid artery stent promote tumor growth? World journal of surgical oncology. 2016;14(1):32.

11. Ebi J, Sato H, Nakajima M, et al. Incidence of leukoencephalopathy after whole-brain radiation therapy for brain metastases. International journal of radiation oncology, biology, physics. 2013;85(5):1212-1217.

12. Wu J, Langford LA, Schellingerhout D, et al. Progressive multifocal leukoencephalopathy in a patient with glioblastoma. Journal of neuro-oncology. 2011;103(3):791-796.

13. Montemurro N, Anania Y, CagnazzoF, et al. Survival outcomes in patients with recurrent glioblastoma treated with Laser Interstitial Thermal Therapy (LITT): A systematic review. Clinical neurology and neurosurgery. 2020;195:105942.

14. KarachiA, Dastmalchi F, Mitchell DA, et al. Temozolomide for immunomodulation in the treatment of glioblastoma. Neuro-oncology. 2018;20(12):1566-1572.

15. Brandes AA, Tosoni A, Franceschi E, et al. Recurrence pattern after temozolomide concomitant with and adjuvant to radiotherapy in newly diagnosed patients with glioblastoma: correlation With MGMT promoter methylation status. Journal of clinical oncology: official journal of the American Society of Clinical Oncology. 2009;27(8):1275-1279.

16. Huang YK, Lieu AS. Treatment response of bevacizumab combination chemotherapy in recurrent glioblastoma: A long-term retrospective study in Taiwan. Medicine. 2020;99(8):e19226.

17. Kim MM, UmemuraY, Leung D. Bevacizumab and Glioblastoma: Past, Present, and Future Directions. Cancer journal. 2018;24(4):180-186.

18. Brown NF, Carter TJ, Ottaviani D, et al. Harnessing the immune system in glioblastoma. British journal of cancer. 2018;119(10):1171-1181.

19. Soffietti R, Ahluwalia M, Lin N, et al. Management of brain metastases according to molecular subtypes. Nature reviews Neurology. 2020;16(10):557-574. 
20. Anton S, Margold M, Kowalski T, et al. Complications of intracerebroventricular chemotherapy via subgaleal reservoir in primary central nervous system lymphoma: A single-institution experience on 1247 installations in 94 consecutive patients. Hematological oncology, 2021;39(2):176-184.

21. Kwakman, J, Elshot YS, Punt C, et al. Management of cytotoxic chemotherapy-induced hand-foot syndrome. Oncology reviews. 2020;14(1):442.

22. Tomoto $\mathrm{K}$, Fujimoto A, Inenaga $\mathrm{C}$, et al. Experience using mTOR inhibitors for subependymal giant cell astrocytoma in tuberous sclerosis complex at a single facility. BMC neurology. 2021;21(1):139.

23. Nabors LB, Supko JG, Rosenfeld M, et al. New Approaches to Brain Tumor Therapy (NABTT) CNS Consortium. Phase I trial of sorafenib in patients with recurrent or progressive malignant glioma. Neuro-oncology. 2011;13(12):1324-1330.

24. Raub TJ, Wishart GN, Kulanthaivel P, et al. Brain Exposure of Two Selective Dual CDK4 and CDK6 Inhibitors and the Antitumor Activity of CDK4 and CDK6 Inhibition in Combination with Temozolomide in an Intracranial Glioblastoma Xenograft. Drug metabolism and disposition: the biological fate of chemicals. 2015;43(9):1360-1371.

25. Hygino da Cruz LC, Jr Rodriguez I, Domingues RC, et al. Pseudoprogression and pseudoresponse: imaging challenges in the assessment of posttreatment glioma. AJNR. 2011;32(11):1978-1985.

26. Batchelor TT, Sorensen AG, di Tomaso E, et al. AZD2171, a pan-VEGF receptor tyrosine kinase inhibitor, normalizes tumor vasculature and alleviates edema in glioblastoma patients. Cancer cell. 2007;11(1):8395
27. Akhtar S, Chaturbedi A. Current and Future Perspective of Glioblastoma, Molecular Heterogeneity with Clinical case. Neurol Neurosurg Curr Res. 2021;2(1):12-18.

28. Parsons DW, Jones S, Zhang X, et al. An integrated genomic analysis of human glioblastoma multiforme. Science. 2008;321(5897):18071812.

29. Hartmann C, Hentschel B, Wick W, et al. Patients with IDH1 wild type anaplastic astrocytomas exhibit worse prognosis than IDH1-mutated glioblastomas, and IDH1 mutation status accounts for the unfavorable prognostic effect of higher age: implications for classification of gliomas. Acta neuropathologica. 2010;120(6):707-718.

30. Hegi ME, Diserens AC, Gorlia T, et al. MGMT gene silencing and benefit from temozolomide in glioblastoma. The New England journal of medicine. 2005;352(10):997-1003.

31. Brat DJ, Verhaak RG, Aldape KD, et al. Comprehensive, Integrative Genomic Analysis of Diffuse Lower-Grade Gliomas. The New England journal of medicine. 2015;372(26):2481-2498.

32. Eskilsson E, Rosland GV, Solecki G, et al. EGFR heterogeneity and implications for therapeutic intervention in glioblastoma. Neuro-oncology. 2018;20(6):743-752.

33. Alemany M, Velasco R, Simo M, et al. Late effects of cancer treatment: consequences for long-term brain cancer survivors. Neuro-oncology practice. 2020;8(1):18-30.

34. Jovcevska I. Genetic secrets of long-term glioblastoma survivors. Bosnian journal of basic medical sciences. 2019;19(2):116-124. 